\title{
IS THERE A LINK BETWEEN SPINE AND HIP MOBILITY?
}

\author{
Miroslav Savič ${ }^{*}$ and \\ S2P, Laboratory for Motor Control and Motor Learning, Ljubljana, Slovenia \\ Nejc Sarabon \\ S2P, Laboratory for Motor Control and Motor Learning, Ljubljana, Slovenia \\ Science and Research Centre Koper, Institute for Kinesiology Research, University of \\ Primorska, Koper, Slovenia
}

\begin{abstract}
Measurement of spinal range of motion is frequently used objective approach in assessment of patients with low back pain, also because of the ease of use. Although emphasized, stretching of hip flexors and extensors is often performed inappropriately. We hypothesized that subjects with reduced hip mobility will probably compensate with pronounced spine mobility and that a significant negative correlation exist between these two entities. Fifty one healthy adults (age $43.7 \pm 15.1$ years) that are regularly involved in moderate physical activity (agility and endurance) participated in this study. Range of motion was measured by means of goniometry and adjusted Schober method that were previously shown to be reliable methods for spine and hip mobility assessment. The correlation between spine movements in different anatomical planes and correlation between spine and hip mobility was calculated. Pearson correlation coefficients were calculated between pairs of flexibility variables. Contrary to our expectations, analysis revealed absence of the correlation between the flexion of the trunk and flexion of the hip. However, moderate correlations were found between flexibility parameters related to trunk movements in different direction. Ranges of motion of the hip and of the trunk give complementary information and cannot be predicted from one another. Therefore, mobility of both joints/regions should be evaluated in order to get insightful information about movement function of the lumbo-pelvic region either in the context of low back pain or sports performance.
\end{abstract}

Keywords: range of motion, trunk, hip, correlation

\section{Introduction}

Measurements of hip and spinal range of motion are most frequently used objective measures in rehabilitation and prevention of the trunk musculoskeletal health problems. It has

\footnotetext{
* Corresponding author. S2P Ltd, Laboratory for Motor Control and Motor Learning, Tehnološki park 19, SI-1000, Ljubljana, Slovenia, e-mail: miroslav.savic@s2p.si
} 
been shown that hamstring`s flexibility is reduced in lower back pain (LBP) patients in comparison to healthy subjects (Johnson \& Thomas, 2010). Mayer, Tencer, Kristoferson, and Mooney (1984) reported that subjects with LBP had less overall flexion and that the percentage of lumbar flexion to overall flexion compared to the subjects without LBP.

Forward bending has been clearly recognized as a risk factor for the development ofLBP. Altered movement patterns of the lumbar spine and hips during forward bending may help explain why forward banding is a risk factor for the development of LBP. Shorter hamstrings might influence the lumbo-pelvic rhythm during forward bending and consequently predispose subject to LBP. McGill (2007) have shown that increased lumbar flexion during forward bending tasks increases anterior shear forces on the spine and increases risk of an injury. Thus, lack of the hamstring`s flexibility can lead to increased lumbar flexion of the trunk during forward bending tasks which can increase the risk of an injury of the spine from mechanical stress.

Results of previous studies on the relationship between hip and spinal range of motion are inconsistent. Negative correlation was shown between hamstrings` flexibility and lumbar excursion during the forward reaching task to the low target in healthy population (Johnson \& Thomas, 2010). Those findings are in agreement with theory that increased hamstrings` flexibility decreases the amount of lumbar flexion required during forward reaching. Li, McClure, and Pratt (1996) did not found correlation between the length of the hamstring muscles and lumbar lordosis or pelvic tilt in relaxed standing. They also investigated the influence of hamstring muscles length on the amount of pelvic and lumbar motion during forward banding and found increased hip motion and decreased ratio of lumbar to hip motion during forward bending as hamstring muscle length increased. Stretching of hamstrings did increase hip motion but did not cause less lumbar motion during forward bending. Although some trend toward reduced lumbar motion during initial part of forward bending was observed, the change was not statistically significant.

Therefore the goal of this study was to assess relationship between hip and spine range of motion. We hypothesized that there will be medium-to-high negative correlation between hip and spine range of motion, because the subjects with shortened hamstrings and thus reduced hip mobility may have to compensate with increased spine mobility in order to perform functional activities of everyday living. Additionally, correlation between mobility of lumbar and toracolumbar parts of spine was calculated to evaluate if only partial measures of spine mobility can be representative for total spine mobility in healthy population.

\section{Method}

\section{Subjects}

Fifty-one healthy adults (age $43.7 \pm 15.1$ years, body height $169.9 \pm 9.5 \mathrm{~cm}$, and body weight $72.8 \pm 33.4 \mathrm{~kg}$ )that are regularly involved in moderate physical activity (sport games and cyclic endurance activities) participated in this study. Neither of the participants had a history of neurological diseases, major orthopedic lesions, vestibular or visual disturbance. The interview, during which the details of the study were presented, was carried out prior to the start of the experiment. After explaining the purpose and potential risks of the study, a written informed consent was obtained. The study was approved by the National Medical Ethics Committee.

\section{Procedure and materials}

Subjects first conducted a 5-minute standardized warm-up. Hamstrings` flexibility was then assessed with the straight leg raise manoeuvre (Figure 1-D) using bubble inclinometer (Fabrication enterprises inc., New York, USA). Subjects were supine lying and the contralateral 
leg was fixated parallel with the table $\left(0^{\circ}\right)$. Hip flexion range of motion was initially checked with knee bent to exclude possible restriction that would limit evaluation of hamstring flexibility. Hip extension flexibility was assessed in prone position also using a bubble inclinometer. Investigator manually fixated subject's pelvis by pressing over ipsilateral iliac bone. Hip extension was performed passively over distal thigh with knee flexed $\sim 80^{\circ}$. Spinal mobility was assessed with the use of adjusted Schober method (Figure 1-A) that was previously shown to be a reliable method for spine mobility assessment (Fitzgerald, Wynveen, Rheault, \& Rothschild, 1983). Lumbar flexion mobility was expressed as the difference between the distance from most cranial border of sacrum to spinosus process of the first lumbar vertebrae in relaxed standing and the same distance in full forward bending position. Similarly, toraco-lumbar flexibility was expressed as the difference between distances from most cranial border of sacrum to spinosus process of seventh cervical vertebrae in the same positions. Side flexion flexibility was assessed in barefooted standing position with pelvis fixated (Figure 1-B). Subjects performed full active side flexion of the spine and distance from the floor to the tip of the middle finger was measured. Trunk rotational range of spine motion was assessed in sitting position. Pelvis was fixated to the sitting surface of the custom made chair with the rotating back support which enabled fixation of the shoulders (Figure 1-C). Data from the potentiometer built into the rotational axis of the chair was sampled at $100 \mathrm{~Hz}$ and stored on a PC for later quantification. All flexibility measurements were performed three times and the mean value was used for further analysis. Subjects were tested by the same staff using the same measurement equipment.

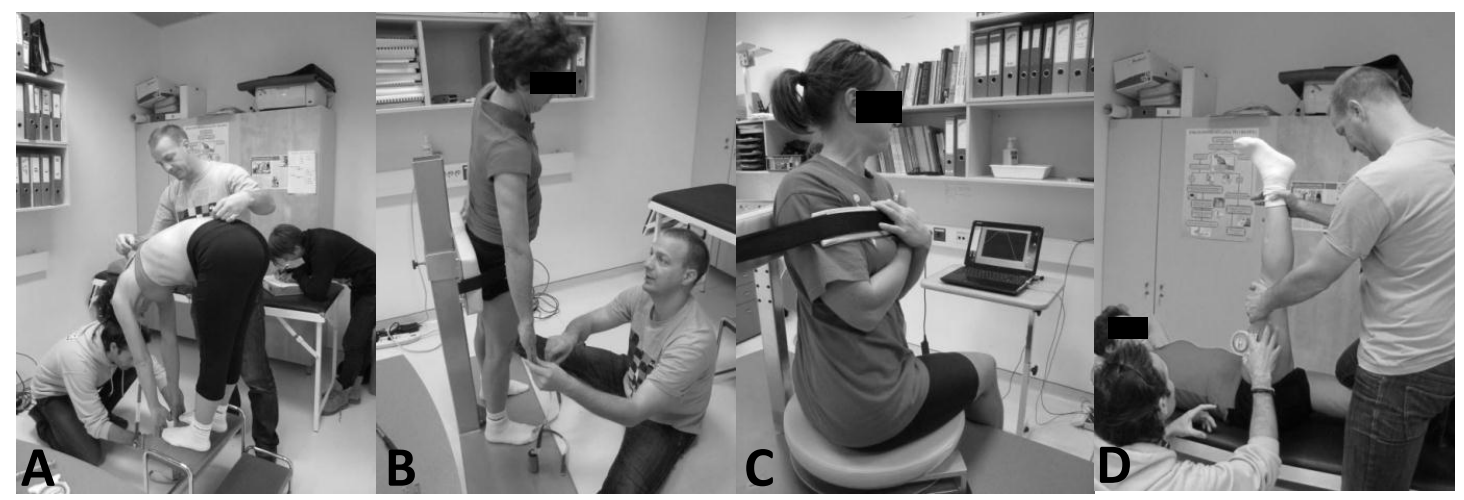

Figure 1. Assessment of spinal flexion mobility with adjusted Schober method (A); Assessment of spinal side flexion (B) and rotational (C) mobility; Assessment of hip flexion mobility (D).

\section{Statistical analysis}

For statistical analyses, SPSS 18.0 software (SPSS Inc., Chicago, USA) was used. Descriptive statistics were calculated for all measured variables. Normality of data distribution was confirmed using Shapiro-Wilk test. Pearson's correlation coefficient was then calculated between different planes of spine movement. Pearson's correlation coefficient was also calculated between spine flexion and hip flexion. In all analysis, a probability less than 0.05 was considered statistically significant.

\section{Results}

Correlations between the flexion of the trunk and flexion of the hip are shown in Figure 2 $(a, b)$ and correlation between different direction related to the trunk movements is shown in Figure 2 (c). The analysis revealed low correlation between the trunk flexion and hip flexion $\left(0.002<\mathrm{R}^{2}<0.004\right)$, which is contrary to our hypothesis, while medium correlation between 
flexibility parameters related to trunk movements in different direction is revealed $\left(0.4<\mathrm{R}^{2}<\right.$ $0.5)$.

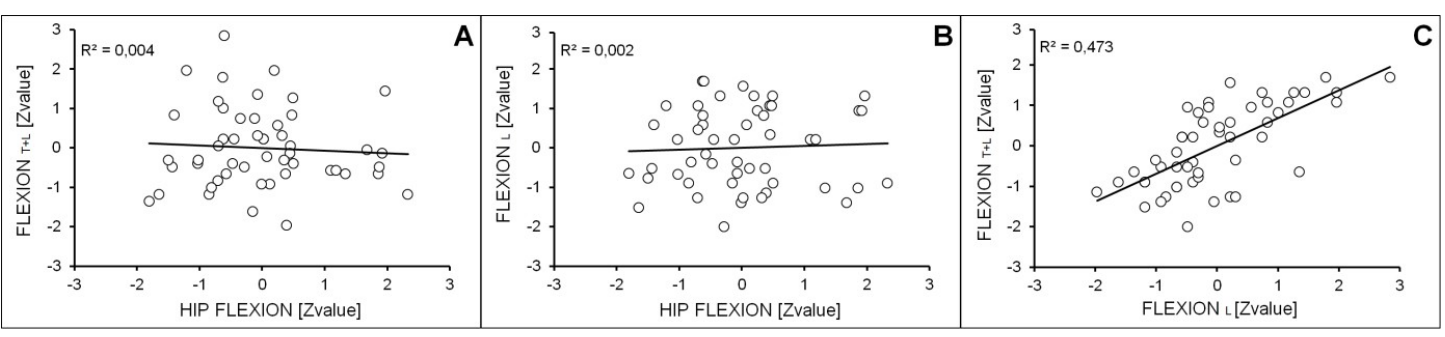

Figure 2. Scater plot ilustrating the correlations $\left(\mathrm{R}^{2}\right)$ between different range of motions

\section{Discussion}

Many of the tasks that occur during either work or everyday activity require forward bending, which is a complex combination of lumbar and hip movement. Therefore, the goal of this study was to assess relation between hip and spine range of motion in healthy individuals. In contrast to some previous reports, our results indicate the absence of correlation between the hamstring and trunk flexibility.

Findings are in agreement with those of Esola, McClure, Fitzgerald, and Siegler (1996) who analysed lumbar and hip motion during forward bending in subject with and without LBP. No difference was observed in total contribution of the lumbar and hip motion in full forward banding. However, there were differences in the pattern of the motion. Contribution of lumbar motion in the first $30^{\circ}$ of forward bending was higher in LBP group which showed that subjects with LBP tend to expend their available lumbar spine motion earlier during the forward bending.

Toppenberg and Bullock (1998) examined the relationships between spinal curvatures, pelvic tilt and lengths of different surrounding muscles (abdominal, erector spinae, iliopsoas, gluteal rectusfemoris and hamstring muscles) in the relaxed standing posture. They found no correlation between pelvic tilt and lumbar curvature. On the other hand, longer abdominal muscles and shorter erector spinae muscles were associated with increased lumbar lordosis. Also the length of the hamstring muscles was negatively correlated to the lumbar curve, meaning that shorter hamstrings were associated with a greater degree of lumbar lordosis (Toppenberg \& Bullock, 1998).This correlation is somewhat surprising and is probably consequence of other muscles influencing spine curvature in standing position since there is no tightening of hamstrings in this position.

Results of this study are in contrast with hypothesis that reduced hamstring flexibility will be correlated with increased spine mobility. Although restricted hip mobility has been previously shown to influence the lumbo-pelvic rhythm (Johnson \& Thomas, 2010), there is no correlation with total spine mobility. One of the reasons may be that participants in this study were healthy individuals with no LBP and with no known restrictions in hamstrings` flexibility. Although correlation between lumbar and thoraco-lumbar mobility was significant, it was very low. In order to get representative information of spine mobility separate assessment of lumbar and thoraco-lumbar spine would be recommended as these deliver different/complementary (and not correlated) information. 


\section{Acknowledgment}

Nejc Sarabon would like to acknowledge the support of the Slovenian Research Agency, grant no. L5-4293.

\section{References}

Esola, M. A., McClure, P. W., Fitzgerald, G. K., \& Siegler, S. (1996). Analysis of lumbar spine and hip motion during forward bending in subjects with and without a history of low back pain. Spine (Phila Pa 1976), 21(1), 71-78.

Fitzgerald, G. K., Wynveen, K. J., Rheault, W., \& Rothschild, B. (1983). Objective assessment with establishment of normal values for lumbar spinal range of motion. Physical Therapy, 63(11), 1776-1781.

Johnson, E. N., \& Thomas, J. S. (2010). Effect of hamstring flexibility on hip and lumbar spine joint excursions during forward-reaching tasks in participants with and without low back pain. Archives of Physical Medicine and Rehabilitation, 91(7), 1140-1142.

Li, Y., McClure, P. W., \& Pratt, N. (1996). The effect of hamstring muscle stretching on standing posture and on lumbar and hip motions during forward bending. Physical Therapy, 76(8), 836-845.

Mayer, T. G., Tencer, A. F., Kristoferson, S., \& Mooney, V. (1984). Use of noninvasive techniques for quantification of spinal range-of-motion in normal subjects and chronic low-back dysfunction patients. Spine, 9(6), 588-595.

McGill, S. (2007). Low Back Disorders: Evidenced-Based Prevention and Rehabilitation. Human Kinetics.

Toppenberg, R. M., \& Bullock, M. I. (1998). The interrelation of spinal curves, pelvic tilt and muscle lenghts in the adolescent female. Australian Journal of Physiotherapy, 32(1), 612. 